\title{
Bearing Fault Diagnosis Based on Improved Hilbert-Huang Transform
}

\author{
Fenglei Ma ${ }^{1, a^{*}}$, Xiaoshuai Chen ${ }^{2, b}$, Jilong $\mathrm{Du}^{3, \mathrm{c}}$ \\ Changchun University of Technology, Changchun 130012, P. R. China

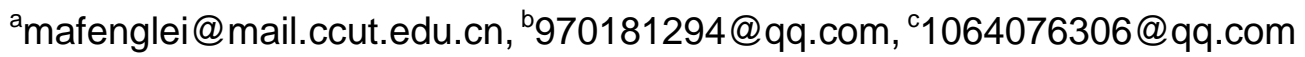

\section{Keywords: Hilbert-Huang transform(HHT);bearing; fault diagnosis}

Abstract. For empirical mode decomposition (EMD) method had endpoint effect and modal aliasing problems, in this paper an improved Hilbert-Huang transform (HHT) based on combination of symmetrical-mirror continuation and correlation coefficient method was put forward. This method can eliminate the modal aliasing and false intrinsic mode function (IMF) problems effectively. Through correction parameters threshold denoising method and the method of selecting sensitive IMF, the Hilbert spectrum can be more accurate for fault diagnosis. The experiment research confirmed that this method can diagnose the bearing fault signal reasons effectively. A new and effective method was proved for bearing fault diagnosis.

\section{Introduction}

Bearing as one of the important parts of internal combustion engine is widely used in petroleum, chemical industry and many other devices, and it is the vulnerable parts in the mechanical equipment and vehicles. For automobile engine, bearing fault will cause irreversible damage to the engine. If the bearing damage will cause the related parts of wear and tear, produce bearing ring, and if the bearing damage seriously, the engine spindle will scrap, cause serious traffic accident ${ }^{[1-2]}$. Therefore, the engine bearing damage must be found as early as possible, in order to avoid unnecessary personnel and property losses.

At present, for the discoveries of faults mainly rely on the driver's experience and the solutions to the main basis is the size of the bearing shell to produce sound voice ${ }^{[3-4]}$. The size of the louder sound as basis on the solution of the problem has contingency and unreliability, for the solution of the problem with great difficulty. Aiming at the shortcomings of the above methods, this paper proposes an improved Hilbert-Huang transform method. The improved Hilbert-Huang transform method can overcome endpoint effect and modal aliasing problems effectively, using this method to fault diagnosis the bearing fault signals, prove a new and effective method for this problem.

\section{Hilbert-Huang transform}

HHT is an analytical method to deal with non-linear and non-stationary signals. It consists of empirical mode decomposition (EMD) and Hilbert analysis ${ }^{[5-6]}$.

\section{Empirical mode decomposition}

EMD can be arbitrarily complex signal decomposition, according to the following steps to get IMF:

To find all local value points in the signal and use cubic spline to make all local maximum points and minimum points together. Formation of upper and lower envelope, calculate the mean of the upper and lower envelope $m_{1}(t)$. Then calculate the original signal $x(t)$ and $m_{1}(t)$, we write it as $h_{1}(t)$. To see whether it meets the IMF conditions or not, if not meet, repeat the above steps, until it meets the IMF's conditions. From these steps, we can get the first IMF component of $x(t)$. Separate $c_{1}(t)$ from $x(t)$ and get the residual quantity $r_{1}(t)$. Using $r_{1}(t)$ to repeat the above process, until can not get IMF, and then the EMD is to stop. By the decomposition of $\mathrm{n}$, the signal of $x(t)$ can write as: 


$$
x(t)=\sum_{k=1}^{n} c_{k}(t)+r_{n}(t)
$$

\section{Hilbert analysis}

Hilbert analysis is mainly to all IMF's to make Hilbert transform, we can get all IMF's instantaneous frequency and amplitude.

Set $\bar{x}(t)$ as the Hilbert transform of signal $x(t), a(t)$ is the instantaneous amplitude and $\theta(t)$ is the instantaneous phase. Can be constructed analytical signal:

$$
z(t)=x(t)+\bar{j} x(t)=a(t) e^{j \theta(t)}
$$

Among them:

$\bar{x}(t)=\frac{1}{\pi} \int_{-\infty}^{\infty} \frac{x(\tau)}{t-\tau} d \tau \quad$, the instantaneous amplitude is $a(t)=\sqrt{x(t)^{2}+\bar{x}(t)^{2}}$ and further instantaneous frequency can be calculated:

$$
f(t)=\frac{\pi}{2} \cdot \frac{d \theta(t)}{d t}
$$

We can get:

$$
x(t)=\operatorname{Re} \sum_{i=1}^{n} a_{i}(t) e^{j \theta_{i}(t)}
$$

On the type of (4), Re represents the real part, the residual amount of $r_{n}$ is omitted here. The type of (4) is called the Hilbert amplitude spectrum, referred to as the Hilbert spectrum.

\section{The Improved EMD}

In the process of the EMD decomposition, because of the uncertainty of the signal endpoint in the extreme, each spline interpolation will cause error, error accumulating make the IMF components have larger error, serious situation will make the decomposition of the data loss of meaning ${ }^{[7]}$. At the same time, because of the discontinuity of signal will cause modal aliasing problem ${ }^{[8]}$. Reduce the effectiveness and accuracy of the HHT method in fault diagnosis.

This paper uses combination of symmetrical-mirror continuation method to Inhibition of the endpoint effect. And adopt the correlation coefficient method to avoid the state aliasing problem ${ }^{[9]}$.

Symmetrical continuation method is optimized by using the endpoint signal on both ends of the continuation of a local extreme value point, to obtain the optimal endpoint values, and use mirror continuation method remove signal endpoint mapping into endless loop signal, to restrain the endpoint effect effectively ${ }^{[10]}$.

\section{Experimental Analysis}

To prove the improved EMD method is effective and accurate, this paper studies the engine sound signal. Using B\&K sound acquisition device, signal acquisition device is mainly composed of engine, sound sensor, data collector and data processor and other parts.

Using sound sensor amplifier engine sound signal, the data collector to collect sound vibration signal, data processor to collect the voice of the vibration signal analysis of HHT. Compared with the vibration signal collected by acceleration sensors, sound acquisition belong to non-contact vibration signal, easy operation, low cost, have the advantage of vibration signal cannot replace ${ }^{[11]}$. 
As shown in figure 1 , for the voice of the normal vibration signals of a car engine, the sampling frequency is $6000 \mathrm{~Hz}$, sampling time is $1.4 \mathrm{~s}$.

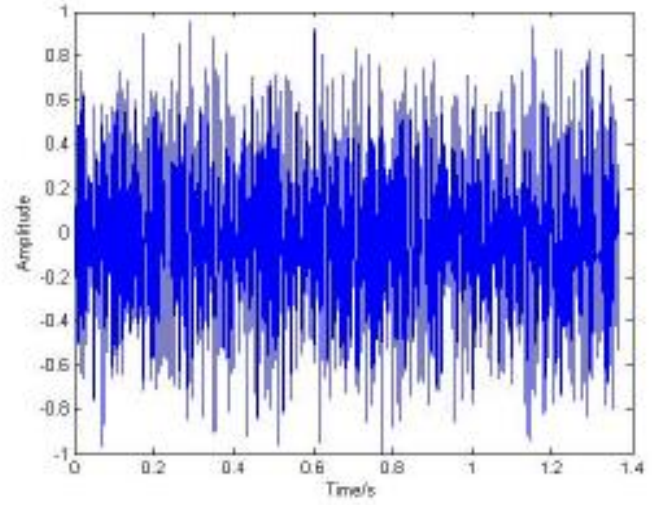

Fig.1 Normal engine signal

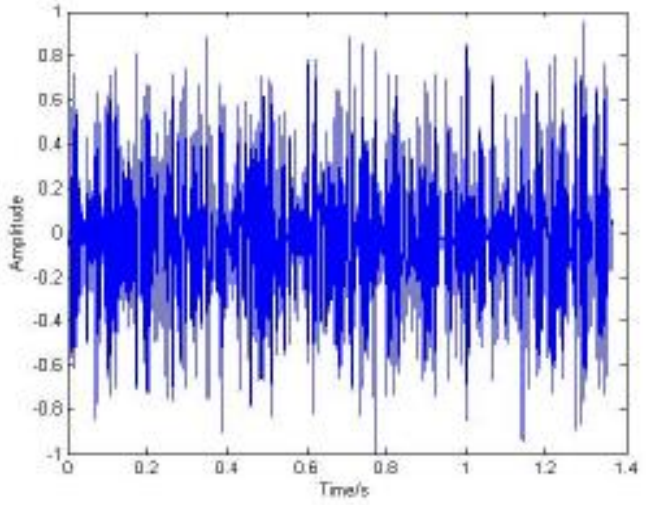

Fig.2 Improved threshold denoising signal

From the figure 1, due to the influence of environmental factors will reduce the signal noise ratio and the HHT analysis accuracy, at the same time increase the difficulty of fault detection. So, use the correction parameters threshold denoising method to reduce the noise ${ }^{[12]}$. After this processing the signal as shown in figure 2. From figure 2, factors such as environmental noise have been improved obviously.

After remove the noise, use traditional EMD and improved EMD method to decompose the signal, get 9 and 13 IMF components. The number of the IMF component reflects the improve EMD method is much more accuracy then the traditional method.

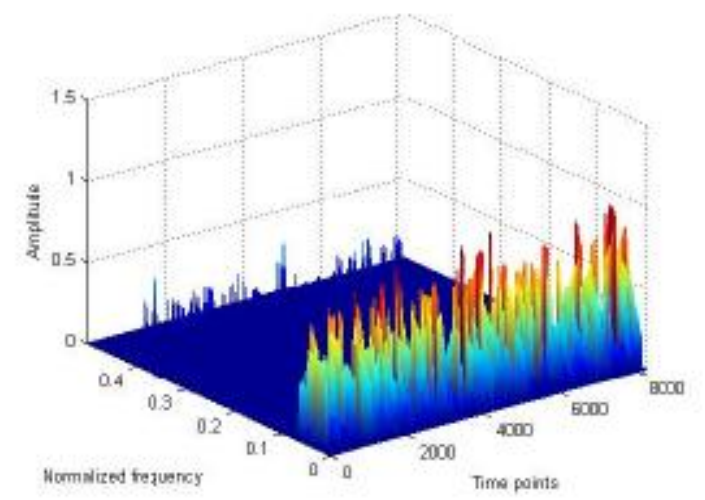

Fig.3 Traditional Hilbert spectrum

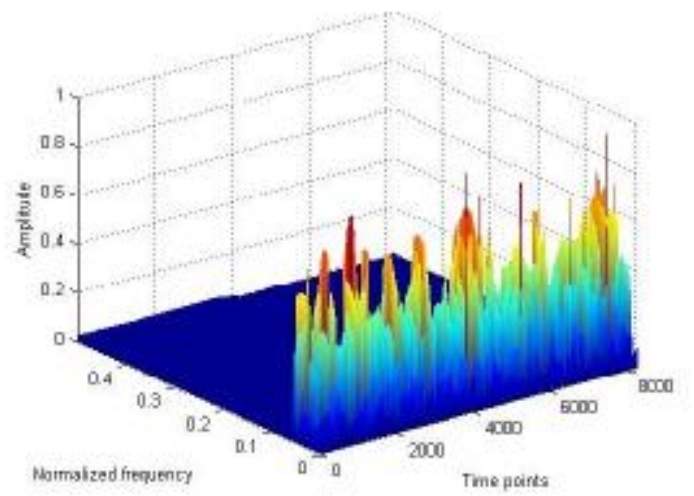

Fig.4 Improvement of the Hilbert spectrum

Figure 5 is the fifth IMF component of the traditional EMD and the improved EMD. Among them, the solid line for traditional EMD method, the dashed for the improvement of the EMD method. From figure 5, the fifth IMF component of the traditional EMD near the end, vibration is obvious, to produce distortion phenomenon in the $0.2 \sim 0.5 \mathrm{~s}$. But, the fifth IMF component of the improved EMD does not have this appearance, and distortion phenomenon has also been inhibited significantly, waveform is more symmetrical and uniform. From these analyze, the improved EMD obtain the IMF component more accurate than the traditional EMD method.

\section{Experimental research}

Using the above methods to diagnosis the automobile engine bearing fault signal. Of the main reasons causing the bearing damage is: alloy melt, scratch alloy, alloy, alloy wear, bearing up and so on. The bearing shell channeling move is one of the main cause of bearing damage. Figure 6 is noise reduction sliding bearing signals. The cause of sliding bearing is the crankshaft increase axial clearance, produce axial channeling.

Using the improved EMD method to analysis the signal. The signal after the improved EMD decomposition gets 13 IMF components. Table 1 is $1 \sim 7$ IMF component and the correlation coefficient of the original signal. The EMD decomposition of the IMF component and the original 
signal satisfy orthogonal, so the IMF component should have higher correlation coefficient with the original signal ${ }^{[13]}$. Signal correlation coefficient is higher, the IMF component contains the more physical characteristics, using this IMF component as a sensitive component fault diagnosis, has higher accuracy and efficiency.

\begin{tabular}{|c|c|}
\hline Tab.1 Seven former IMF correlation coefficient \\
\hline IMF Component & Correlation Coefficient \\
\hline IMF1 & 0.9826 \\
\hline IMF2 & 0.8040 \\
\hline IMF3 & 0.4816 \\
\hline IMF4 & 0.2880 \\
\hline IMF5 & 0.0833 \\
\hline IMF6 & 0.0157 \\
\hline IMF7 & 0.0003 \\
\hline
\end{tabular}

From table 1, the 1 4 IMF component is sensitive component, analysis these IMF components by Hilbert, and get 3D Hilbert spectrum as shown in figure 6.

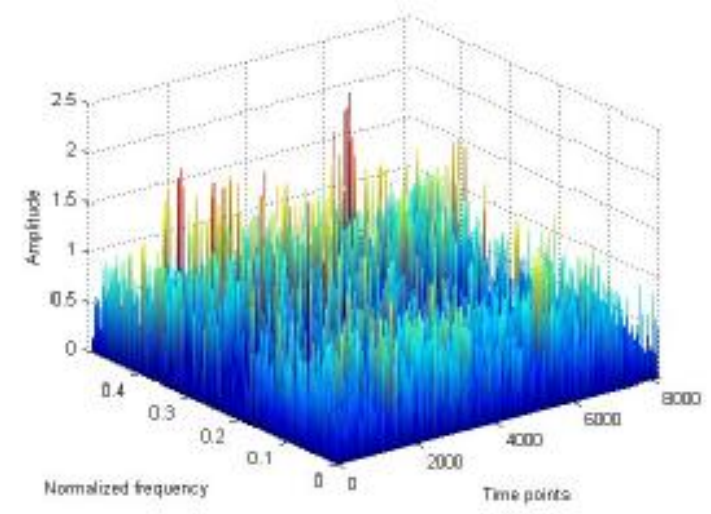

Fig.5 Sensitive IMF component composition the Hilbert spectrum

From figure 6, The Hilbert spectrum extracts the fault feature signals accurately: (1) due to the bush axial motion, engine actual frequency is difficult to measure; (2) no periodic distribution energy; (3) no periodic frequency distribution, energy appear from low to high frequency; (4) the high frequency part have bigger energy random distribution.

Scratch alloy, alloy and wear is the main cause of bearing damage, and they are due to local reasons to cause bearing damage, the characteristics of its frequency in cycles should be enhanced, and energy have cyclical. But bearing up to move is the overall damage of bearing bush, frequency and amplitude should be different from local causes of damage, because nonsocial damage at the same time, frequency and amplitude does not have obvious periodicity. From the characteristics of the Hilbert spectrum analysis can be seen that in the Hilbert spectrum do not have local damage related features. And can accurately judge the bearing fault types for the bearing dynamic and it in conformity with the actual reasons. Therefore, the test result diagnoses the cause of bearing damage effectively.

\section{Conclusions}

Through the test, the conclusions as follows:

(1) Using the improved EMD can solve the endpoint effect and modal aliasing phenomenon of HHT diagnostic accuracy effectively.

(2) According to the characteristics of the sliding bearing fault diagnosis, Through correction parameters threshold denoising method and the method of selecting sensitive IMF, the Hilbert spectrum can be more accurate for fault diagnosis. 
(3) Example analysis shows that this method can extract bearing fault signal characteristics effectively, accurate diagnosis the cause of the problem, and has important use value.

\section{References}

[1] HU Ding-shan, YAO Xiao-xu: The damage reasons and selective fit of engine' s bearing shell [J]. Move Power Station \& Vehicle, 2002, 4: 33-34.

[2] Chen Ying-guo: Troubleshooting for Abnormal Sound of Engine Bearing Pad [J]. Beijing Automobile, 1994,3: 45.

[3] JI Guo-guang: Typical Fault Diagnosis and Elimination of Steyr Truck[J]. Heavy Truck,2004,2:42-43.

[4] YANG Jian-cheng, CHEN Lan-lin, TANG Qiao-bin: With the exclusion of the WD615 series diesel engine bearing abnormal sound judgment [J]. Auto Repair,2002,10: 22.

[5] Huang N E, Shen Z, Long S R, et al. The empirical mode decomposition and the Hilbert spectrum for nonlinear and non-stationary time series analysis [J]. J. Proc. R. Soc. Lond. A, 1998,454: 903-995.

[6] LEI Ya-guo, HE Zheng-jia, ZI Yan-yang: Fault Diagnosis Based on Novel Hybrid Intelligent Model [J]. CHINESE JOURNAL OF MECHANICAL ENGINEERING, 2008, 44(7):112-117.

[7] WANG Chuan-fei, AN Gang, WANG Kai, ea 1t. Improved Method for End Effects of EMD Based on Mirror Extension and Neural Network [J]. Journal of Academy of Armored Force Engineering, 2010,24(2): 62-65.

[8] LEI Ya-guo: Machinery Fault Diagnosis Based on Improved Hilbert-Huang transform [J]. CHINESE JOURNAL OF MECHANICAL ENGINEERING, 2011,47(5): 71-77.

[9] QIAO Xin-yong, ZHAO Xiu-yu, LIU Chun-hua: Approach to Depress the Boundary Effect in EMD Analysis [J]. SOFTWARE, 2012,33(8): 72-74.

[10] WU Z H, HUANG N E: Ensemble empirical mode decomposition: A noise assisted data analysis method [J]. Advances in Adaptive Data Analysis, 2009,1:1-41.

[11] MA Feng-lei, ZHENG Yong-tao, JIANG Zhen-hai: Fault Diagnosis of Bearing Pad Used in Hilbert-Huang Transform [J]. Journal of Changchun University of Technology (Natural Science Edition), 2014,1: 55-59.

[12] MENG Zong, LI Shan-shan: Rolling bearing fault diagnosis based on improved wavelet threshold de-noising method and HHT [J]. JOURNAL OF VIBRATION AND SHOCK, 2013,32(14): 204-208.

[13] XU Bin, XU De-cheng, ZHU Wei-ping, ea lt. Improving Hilbert-Huang Transform(HHT) Method [J]. Journal of Northwestern Polytechnical University, 2011,29(2): 268-272. 